\title{
Evolution of NCoR-1 and NCoR-2 corepressor (1) alternative mRNA splicing in placental mammals
}

\author{
Martin L. Privalsky ${ }^{1 *}$ (1) and Michael L. Goodson ${ }^{2}$
}

\begin{abstract}
Objective: The NCoR-1 and NCoR-2 corepressors are products of an early gene duplication near the beginning of vertebrate evolution and play both overlapping and divergent roles in development and physiology. Alternative-splicing of NCOR-1 and NCOR-2 further customizes their functions. To better understand the evolutionary basis of this phenomenon we extended our prior study of NCoR-1 and NCoR-2 alternative-splicing to an expanded series of species.

Results: Alternative-splicing of NCoR-2 was observed in all vertebrates examined whereas alternative-splicing of NCoR-1 was largely limited to placental mammals. Notably the most prominent of the NCoR-1 alternative-splicing events specific to the placental lineage, in exon 37 that plays a key role in murine metabolism, mimics in many features an analogous alternative-splicing event that appeared in NCoR-2 much earlier at the beginning of the vertebrate radiation. Detection of additional alternative-splicing events, at exons 28 in NCoR-1 or NCoR-2, was limited to the Rodentia or Primates examined, indicating both corepressor paralogs continued to acquire additional splice variations more recently and independently of one another. Our results suggest that the NCoR-1/NCoR-2 paralogs have been subject to a mix of shared and distinct selective pressures, resulting in the pattern of divergent and convergent alternative-splicing observed in extant species.
\end{abstract}

Keywords: Alternative mRNA splicing, Evolution, Corepressors, Transcriptional regulation, Nuclear receptors, Nuclear hormone receptors, NCOR, SMRT

\section{Introduction}

The NCoR-1 and NCoR-2 corepressors bind and mediate transcriptional repression by the nuclear receptor family of hormone-regulated transcription factors [1-5]. NCoR- 1 and -2 share $~ 40 \%$ sequence identity and a common molecular architecture [5-7]. Both NCoR-1 and -2 possess N-terminal Silencing Domains (SDs) that recruit additional proteins to form a larger corepressor holocomplex, and C-terminal Receptor Interaction Domains (RIDs) that tether these holocomplexes to their nuclear receptor partners on target genes [6, 8-11]. Despite these commonalities NCoR-1 and -2 exert unique as well as overlapping molecular and biological functions $[6,8]$.

Whereas NCoR-1 and NCoR-2 are present as two distinct loci in all the craniate genomes analyzed, NCoR

\footnotetext{
*Correspondence: mlprivalsky@ucdavis.edu

${ }^{1}$ Department of Microbiology and Molecular Genetics, College

of Biological Sciences, University of California at Davis, Davis, CA 95616 USA

Full list of author information is available at the end of the article
}

sequences in cephalochordates, urochordates, hemichordates, and echinoderms are encoded by a single locus [12]. NCoR-1 and -2 are therefore considered the products of gene duplication and divergence events that began near the origin of vertebrate evolution. Notably both NCoR-1 and -2 in mammals undergo alternative mRNA splicing that further broadens the diversity of actions available to each paralog and allows these actions to be customized for different purposes in different cell types [12-23], and in response to different signals [24, 25]. Understanding the evolution and extant patterns of utilization of these alternative-splicing events is important for understanding the biological roles of the different splice variants and how the functions of the NCoR paralogs have been customized to fit the differing requirements of different organisms.

We previously reported that whereas NCoR-2 alternative-splicing was detected in all the vertebrates examined, NCoR-1 splicing was apparently restricted to the placental mammals, an observation suggesting these two 
corepressors may have been subject to distinct forms of evolution subsequent to their gene duplication [23]. However our prior study was limited in scope with only 3 placental mammals and 5 non-placental vertebrates analyzed. To better understand the evolutionary relationships in this phenomenon, we have now expanded our analysis of alternative-splicing of NCoR-1 and NCoR-2 to a significantly wider series of eutherian lineages. Our new data help to confirm and elaborate on the concepts we proposed in our prior study, as well as providing a broader information base for further analysis and study.

\section{Main text}

\section{Methods}

Alternative-splicing was quantified from RNA prepared from nucleated peripheral blood and assayed by a reverse transcriptase PCR quantification method employing primers that spanned each alternativelyspliced site $[14,16,17,20,21,23,25]$. The primer sequences and a detailed description and discussion of the sensitivity and accuracy of this method are provided in Additional file 1 . The relative abundance of each alternatively-spliced corepressor variant was calculated as a percentage of the sum of all the alternatively-spliced isoforms produced at that alternativesplice location. The mean and standard deviation of 3 or more experiments for each species were determined. Data from several species analyzed previously [23] are included in Fig. 1 for comparison. NCoR-2 was previously referred to as SMRT (an alternative nomenclature); the exon numbering system here is as in [23].

\section{Results \\ Convergent evolution at a nuclear receptor-specificity determinant within NCoR-1/2 exons $37 / 40$}

Alternative mRNA splicing at a receptor specificity determinant (RID-3) within exon 37 of mammalian NCoR-1 (mapping to exon 40 in NCoR-2) is known to

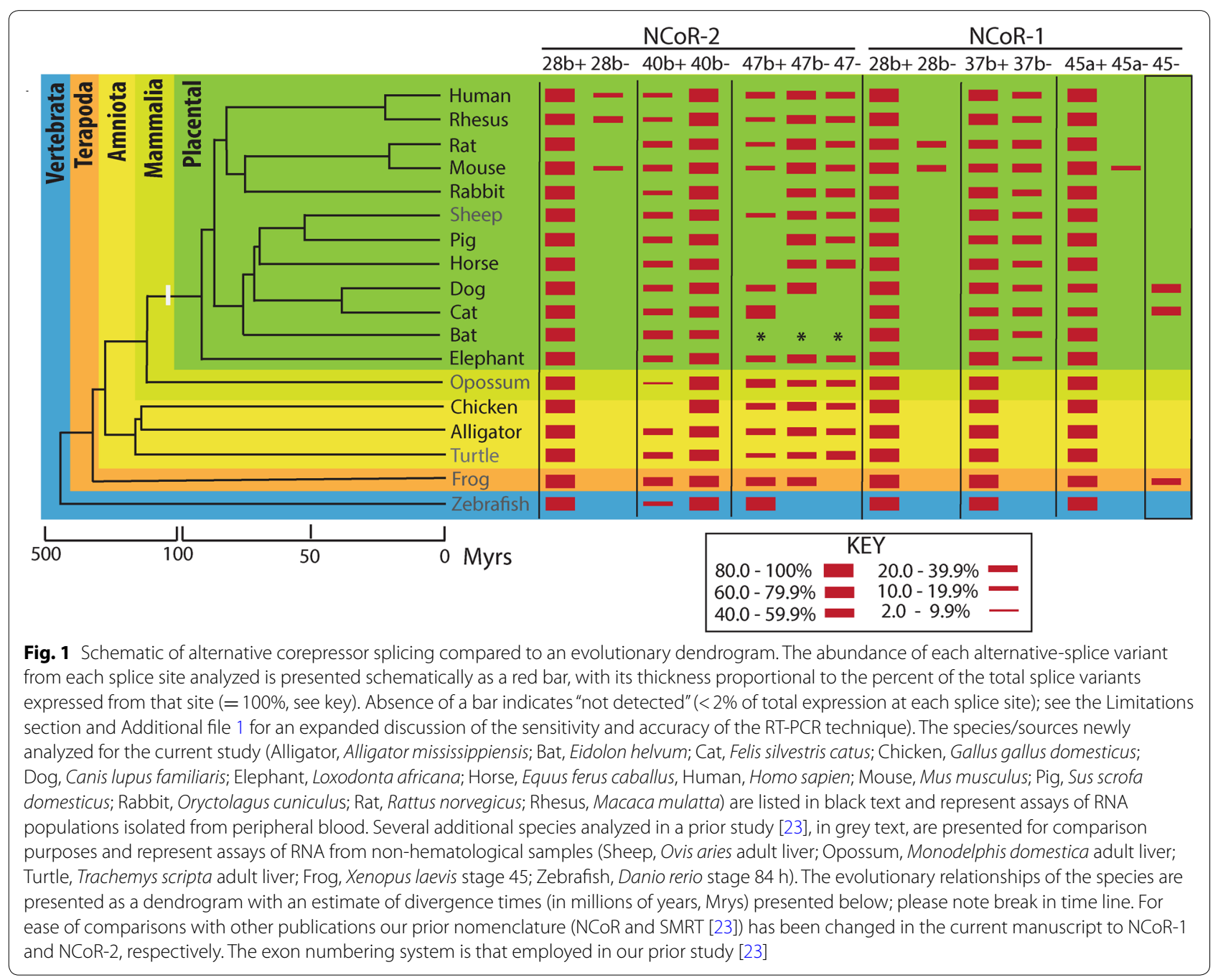


have profound effects on the molecular and biological properties of these corepressors, including the control of lipid and glucose metabolism [13, 16-19]. Our new results confirm that although this alternative-splicing occurred in NCoR-2 in virtually all the vertebrates examined, alternative-splicing at this position in NCoR-1 was limited within our sensitivity of detection to the placental mammals analyzed (Table 1 and Fig. 1). Notably our new results also demonstrated that NCoR-1 exon 37 alternative-splicing occurred in all the placental mammals investigated, including Proboscidea (Table 1 and Fig. 1), indicative of a very early evolutionary acquisition of this trait within this clade and placing this convergent evolutionary event as arising in NCoR-1 300 to 400 million years after the appearance of the comparable alternativesplicing event in NCoR-2.

\section{Divergent evolution at a nuclear receptor-specificity determinant within NCoR-2 exon 47}

Our current results also documented that alternativesplicing at a second site important for nuclear receptorspecificity, within NCoR-2 exon 47 and encompassing/ flanking RID-1 [15], was widely distributed among all the vertebrate lineages examined, but in contrast to the exon $37 / 40$ splice no comparable alternative-splice form was detected in the NCoR-1 paralog in any species tested (Table 1 and Fig. 1). We interpret this alternative-splicing event as a divergent acquisition by the NCoR-2 corepressor early after duplication of the common ancestral gene that, unlike exon $37 / 40$ alternative-splicing, retained a paralog-restricted role over subsequent evolutionary time.

\section{Alternative-splicing events at positions likely to influence functions of the corepressor other than nuclear receptor specificity}

Additional alternative-splicing events were observed in our analysis at NCoR-1 and NCoR-2 exons 28 that were apparently restricted to the Primates and Rodentia examined and were probably acquired relatively late in placental mammalian evolution (Table 1 and Fig. 1). These mapped to or near corepressor regions, such as SD sequences, that are likely to influence assembly or other functions of the corepressor complex rather than nuclear receptor specificity. The precise locations and natures of these alternativesplice sites are non-identical in NCoR-1 versus NCoR-2, consistent with these alternative-spliced also representing the products of divergent evolutionary events.

\section{Newly recognized, taxonomically-restricted alternative-splicing at the $\mathrm{C}$-terminus of NCoR-2}

The current study also newly detected an alternativesplice variant of NCoR-1 that deletes all of exon 45 (exon
45-, Table 1 and Fig. 1), removing portions of a corepressor domain associated with Mitogen-Activated Kinase cascade-regulation [24]. This NCoR-1 splice variant was not seen in NCoR-2 and was only detected in the two Carnivora tested and in an Anura. The latter is notable in that it represents the only observed example of any form of NCoR-1 alternative-splicing in non-mammals. Our failure to previously detect this splice form was a consequence of the unanticipated small size of the corresponding PCR product generated by the primers in our prior studies.

\section{Discussion}

Both NCoR-1 and -2 are subject to alternative mRNA splicing, generating an extensive series of corepressor protein variants that are expressed at different abundances in different tissues, preferentially associate with different transcription factor partners, respond to signal transduction pathways in distinct ways, and play distinguishable, even opposing, biological roles [13, 16-20]. This alternative-splicing is a major determinant of NCoR corepressor function that further broadens the diversity of actions available to each paralog and allows these actions to be customized for different purposes in different cell types and in response to different signals. Understanding and comparing the evolution and extant patterns of utilization of these alternativesplicing events in NCoR-1 versus NCoR-2 should therefore provide important insights into the biological roles of the different splice variants and how the functions of these paralogs have been customized to fit the differing requirements of different organisms.

With this goal we report here an expanded analysis of alternative-splicing in NCoR-1 and NCoR-2 to more fully understand the origins of this phenomenon. Our studies revealed that there are both commonalities and substantial differences in the alternative splicing of NCoR-1 versus NCoR-2, presumably reflecting and helping to mediate the multiple biological roles of these two paralogs.

We previously suggested, based on analysis of a limited number of species, that NCoR-2 alternative-splicing likely occurs in all vertebrates, whereas NCoR-1 splicing is restricted to the placental mammals [23]. Our current expanded study strengthens that hypothesis (with one exception, see below) and given the very early divergence of Afrotheria/Proboscidea during the placental mammal radiation, our new data places the acquisition of NCoR-1 alternative-splicing as likely having occurred during the Cretaceous period some 300 to 400 million years after the acquisition of alternativesplicing by NCoR-2. Further, despite its later evolutionary appearance, the most prominent and wide spread 


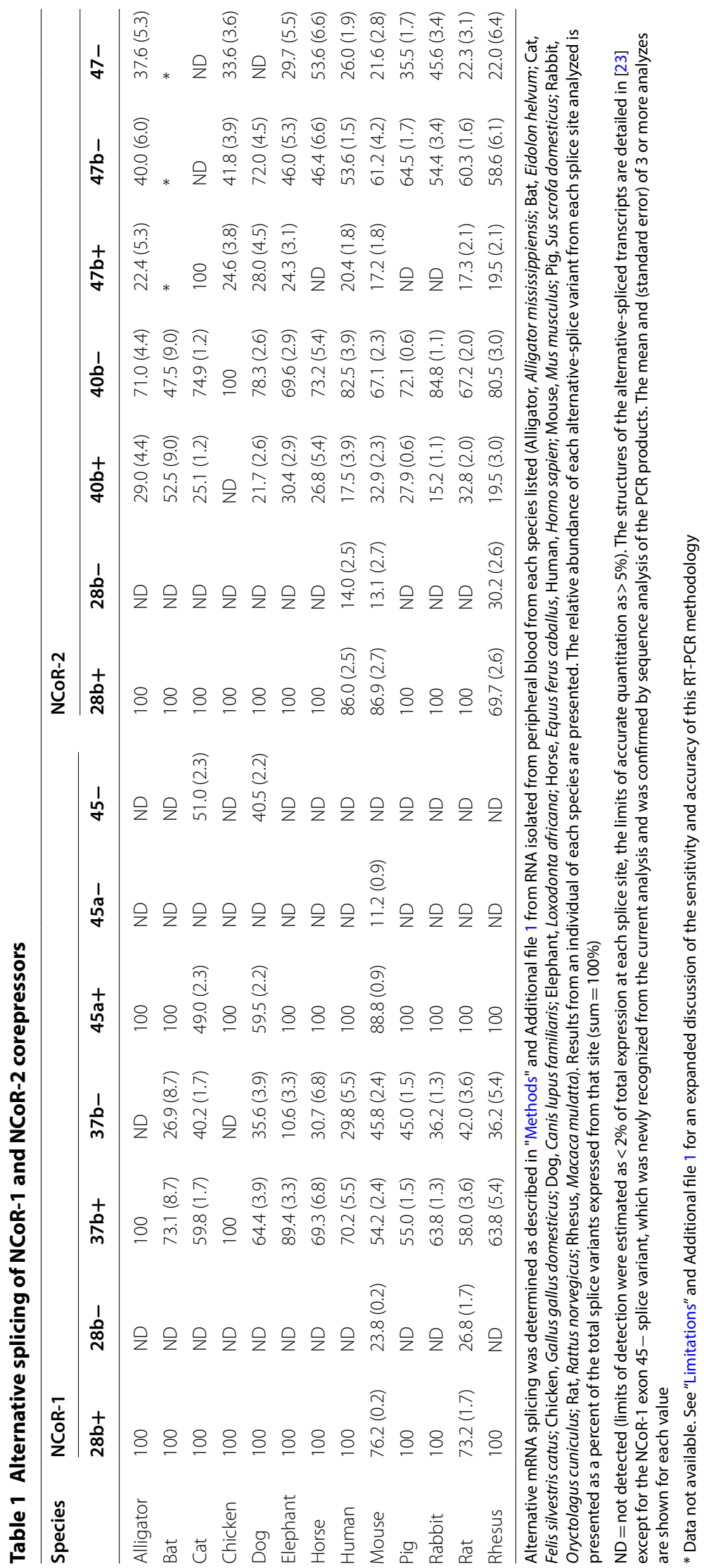


of the alternative-splicing events in mammalian NCoR1 , at the RID-3 domain in exon 37, mimics in many of its structural and molecular features a RID-3/exon 40 alternative-splicing event acquired by NCoR-2 at the beginning of the vertebrate radiation. Alternative-splicing at these sites alters the affinity of the corepressor proteins for their nuclear receptor partners, including those important in control of lipid and carbohydrate metabolism. We speculate that the later acquisition of this alternative-splicing event by NCoR-1 in the placental mammals may reflect a unique role for this corepressor variant in energy homeostasis in this lineage.

Additional alternative-splicing events, at NCoR-1 exon 28 and 45, and at NCoR-2 exon 28 and 47, were each unique to their corresponding paralog and appear to be divergent evolutionary events. The NCoR-2 exon 47 alternative-splice site maps to RID-1 and is known to alter the affinity of the encoded corepressor protein toward a subset of its nuclear receptor partners. In contrast the NCoR-1 exon 28 and NCoR-2 exon 28 splicing events map not to RIDs but to regions likely to influence assembly (SD domains) or other functions of the corepressor complex. These exon 28 splicing events were non-identical in NCoR-1 and NCoR-2 and their detection was limited to the Rodentia and Primates examined, suggesting that both paralogs continued to acquire additional splice variations within the past 90 to 100 million years and did so independently of one another.

Our new analysis also revealed a previously unrecognized alternative-splice event in NCoR-1 exon 45 that removed all of exon 45 and was found exclusively in the two Carnivora tested and in the amphibian Xenopus. The only other alternative-splice event known at this exon removes only a portion of exon 45 [exon 45a-] and is limited to mice. The basis for this tight species restriction is unclear, as are the exact effects of these splicing events on NCoR-1 function, although based on location they may alter regulation of $\mathrm{NCoR}$ function by MAP kinase cascade signaling. The presence of the exon 45- splice in Xenopus is the first known example of NCoR-1 alternative-splicing in a non-mammal. Analysis of additional amphibian and related species will be required to better understand the taxonomic extent, mechanistic origin, and biological implications of this unanticipated observation.

In conclusion our results are most consistent with the NCoR-1 and NCoR-2 corepressor paralogs having been subject to a mix of shared and distinct selective pressures that differed in different lineages, leading to the current pattern of divergent and convergent alternative-splicing events observed in extant species.

\section{Limitations}

This study focuses on alternative-spliced sites within NCoR-1 and -2 that were previously recognized and in many cases established as having molecular or biological effects; there may be additional sites of alternative-splicing in one or more species not queried by the primer pairs used here. Ethical and access limitations in working with many of the species required the current study to be restricted to analysis of peripheral blood samples; we cannot fully exclude the possibility that a splice variant not detected in peripheral blood may nonetheless be expressed by that species in another tissue. Nonetheless our prior studies on model organisms suggest that the detected presence or absence of a given variant in peripheral blood is largely predictive of its detected presence or absence in other tissues in that organism (e.g. [23]). Similarly although our methodology can detect alternative-splice products at levels down to $\sim 2 \%$ of total we cannot exclude certain splice variants were not observed because they are expressed at levels even below this limit; such very low levels of expression, if they exist, would be of unclear biological significance.

Due to practical and ethical limitations it was typically not possible to obtain samples from multiple individuals from within a given (exotic) species; however our analysis of model organisms such as human and mice demonstrated relatively little variation in alternative-splicing from individual to individual (e.g. [14, $16,17,20,23,25]$ and data not shown), indicating that this restriction was unlikely to substantially alter the interpretation of the data presented here. Finally our methodology required access to freshly drawn peripheral blood and sufficient species-specific sequence information to design appropriate PCR primers. These dual requirements restricted our ability to expand our analysis to taxonomies not reported here or to include additional representatives within the lineages that were studied. Nonetheless the patterns of alternative mRNA splicing were generally very similar in the cases where we were able to compare two species within a shared lineage.

\section{Additional file}

Additional file 1. Expanded methods. This file details the RT-PCR methodology, the oligonucleotide primers employed, and the issues relating to the quantitation and sensitivity of the data.

Abbreviations

NCoR: nuclear corepressor; RID: receptor interaction domain; SD: silencing domain. 


\section{Acknowledgements}

Sincere thanks to Kevin P. Bellido, Merritt Clark, Andrea Fascetti, Maria Montano, Kristy N. Portillo, Nicola Pusteria, Robert B. Rebhun, Heather R. Schrader, Caleb Sehnert, Charlie Sammut, Koen K. Van Rompay, and Ray F. Wack for their efforts and generosity in providing biological materials.

\section{Authors' contributions}

MLP performed the experiments, the majority of the analysis, and the bulk of the assembly and writing of this manuscript. MLG pioneered the original methodology, provided essential advice, contributed important insights and valuable interpretations of the data, and assisted in manuscript editing. Both authors read and approved the final manuscript.

\section{Funding}

Funding was through a personal monetary contribution by the corresponding author (MLP) and was administered through the Department of Microbiology and Molecular Genetics, University of California at Davis. Therefore the "funding body" was the corresponding author and contributed to the design of the study, the collection, analysis, and interpretation of the data, and the writing of the manuscript as described for MLP in the authors' contributions section.

\section{Availability of data materials}

All data generated or analyzed during this study are included in this published article

\section{Ethics approval and consent to participate}

All animal use was performed under the guidelines/approval of the University of California at Davis Animal Use and Care Program and Committee (Relevant Protocol $=18834$ ). Samples of human materials used here were anonymouslycoded aliquots that were to be otherwise discarded; i.e. obtained from existing specimens collected under appropriate written informed consent agreements approved by the University of California at Davis's Internal Review Board. As a result our component of the study using these coded aliquots was determined by the UCD IRB to be exempt from formal review under the 46.1019(b)-5 standard ("research involving the collection or study of existing data, documents, records, pathological specimens, or diagnostic specimens, if these sources are publicly available or if the information is recorded by the investigator in such a manner that subjects cannot be identified, directly or through identifiers linked to the subjects"). None of the data provided here relate to health outcomes, nor do they contain identifiers or other information than could be employed to identify the individuals providing the samples.

\section{Consent for publication}

Not applicable. As noted above none of the data provided here contain identifiers or other information than can be employed to identify the individuals providing the samples.

\section{Competing interests}

The authors declare that they have no competing interests.

\section{Author details}

${ }^{1}$ Department of Microbiology and Molecular Genetics, College of Biological Sciences, University of California at Davis, Davis, CA 95616, USA. ${ }^{2}$ Department of Anatomy, Physiology, and Cell Biology, College of Veterinary Medicine, University of California at Davis, Davis, CA 95616, USA.

Received: 22 May 2019 Accepted: 11 June 2019

Published online: 17 June 2019

\section{References}

1. Astapova I, Hollenberg AN. The in vivo role of nuclear receptor corepressors in thyroid hormone action. Biochim Biophys Acta. 2013;1830(7):3876-81.

2. Edwards DP. The role of coactivators and corepressors in the biology and mechanism of action of steroid hormone receptors. J Mammary Gland Biol Neoplasia. 2000;5(3):307-24.

3. Feige JN, Auwerx J. Transcriptional coregulators in the control of energy homeostasis. Trends Cell Biol. 2007;17(6):292-301.
4. Jepsen K, Rosenfeld MG. Biological roles and mechanistic actions of corepressor complexes. J Cell Sci. 2002;115:689-98.

5. Lazar MA. Nuclear receptor corepressors. Nucl Recept Signal. 2003;1:e001.

6. Mottis A, Mouchiroud L, Auwerx J. Emerging roles of the corepressors NCoR1 and SMRT in homeostasis. Genes Dev. 2013;27(8):819-35.

7. Ordentlich P, Downes M, Evans RM. Corepressors and nuclear hormone receptor function. Curr Top Microbiol Immunol. 2001;254:101-16.

8. Stanya KJ, Kao HY. New insights into the functions and regulation of the transcriptional corepressors SMRT and N-CoR. Cell Div. 2009;4:7.

9. Perissi V, Jepsen K, Glass CK, et al. Deconstructing repression: evolving models of co-repressor action. Nat Rev Genet. 2010;11(2):109-23.

10. Privalsky ML. The role of corepressors in transcriptional regulation by nuclear hormone receptors. Annu Rev Physiol. 2004;66:315-60.

11. Watson PJ, Fairall L, Schwabe JW. Nuclear hormone receptor co-repressors: structure and function. Mol Cell Endocrinol. 2012;348(2):440-9.

12. Short S, Peterkin T, Guille $M$, et al. Short linear motif acquisition, exon formation, and alterntaive splicing form a pathway to diversity for NCoR family co-repressors. Open Biol. 2015;5:150063.

13. Faist $F$, Short $S$, Kneale $G G$, et al. Alternative splicing determines the interaction of SMRT isoforms with nuclear receptor-DNA complexes. Biosci Rep. 2009;29(3):143-9.

14. Goodson M, Jonas BA, Privalsky MA. Corepressors: custom tailoring and alterations while you wait. Nucl Recept Signal. 2005;3:e003.

15. Goodson ML, Jonas BA, Privalsky ML. Alternative mRNA splicing of SMRT creates functional diversity by generating corepressor isoforms with different affinities for different nuclear receptors. J Biol Chem. 2005;280(9):7493-503.

16. Goodson ML, Mengeling BJ, Jonas BA, et al. Alternative mRNA splicing of corepressors generates variants that play opposing roles in adipocyte differentiation. J Biol Chem. 2011;286(52):44988-99.

17. Goodson ML, Young BM, Snyder CA, et al. Alteration of NCoR corepressor splicing in mice causes increased body weight and hepatic steatosis without glucose intolerance. Mol Cell Biol. 2014;34:4104-14.

18. Malartre $M$, Short $S$, Sharpe $C$. Alternative splicing generates multiple SMRT transcripts encoding conserved repressor domains linked to variable transcription factor interaction domains. Nucleic Acids Res. 2004;32(15):4676-86.

19. Malartre M, Short S, Sharpe C. Xenopus embryos lacking specific isoforms of the corepressor SMRT develop abnormal heads. Dev Biol. 2006:292(2):333-43.

20. Mengeling BJ, Phan TQ, Goodson ML, et al. Aberrant corepressor interactions implicated in PML-RAR(alpha) and PLZF-RAR(alpha) leukemogenesis reflect an altered recruitment and release of specific NCoR and SMRT splice variants. J Biol Chem. 2011;286(6):4236-47.

21. Mengeling BJ, Goodson ML, Bourguet W, et al. SMRTepsilon, a corepressor variant, interacts with a restricted subset of nuclear receptors, including the retinoic acid receptors alpha and beta. Mol Cell Endocrinol. 2012;351(2):306-16.

22. Short S, Malartre M, Sharpe C. SMRT has tissue-specific isoform profiles that include a form containing one CoRNR box. Biochem Biophys Res Commun. 2005:334(3):845-52.

23. Privalsky ML, Snyder CA, Goodson ML. Corepressor diversification by alternative mRNA splicing is species specific. BMC Evol Biol. 2016;16(1):221.

24. Jonas BA, Varlakhanova N, Hayakawa F, et al. Response of SMRT (silencing mediator of retinoic acid and thyroid hormone receptor) and $\mathrm{N}-\mathrm{CoR}$ (nuclear receptor corepressor) corepressors to mitogen-activated protein kinase kinase kinase cascades is determined by alternative mRNA splicing. Mol Endocrinol. 2007;21(8):1924-39.

25. Snyder CA, Goodson ML, Schroeder AC, et al. Regulation of corepressor alternative mRNA splicing by hormonal and metabolic signaling. Mol Cell Endocrinol. 2015;413:228-35.

\section{Publisher's Note}

Springer Nature remains neutral with regard to jurisdictional claims in published maps and institutional affiliations. 